\title{
La Economía de Género: Las Pensiones Alimenticias y su Relación con la Paternidad y los Derechos Humanos de las Mujeres
}

\author{
The Gender Economy: Alimony and its Relationship with \\ Paternity and Women's Human Rights
}

\author{
Roxana Arroyo * \\ Universidad de Posgrado de Ecuador, Ecuador
}

\begin{abstract}
El presente artículo indaga, de una manera crítica, la institución jurídica de las pensiones alimenticias y su impacto en la economía de las mujeres, lo que la constituye en una suerte de "Violencia Patrimonial" como lo contempla la Convención Interamericana para Prevenir, Sancionar y Erradicar la Violencia contra las Mujeres. Se plantea la necesidad de incorporar el análisis económico del derecho, dentro del cual el fenómeno jurídico aborda la necesidad de examinar los componentes legales, pero además las cadenas de valor desde la perspectiva de género. Se abordan los patrones socioculturales que profundizan y naturalizan las paternidades irresponsables y sus consecuencias en la niñez y la adolescencia en lo relativo a la disminución de recursos económicos para cubrir la educación de su prole y la ausencia de la coeducación, tanto en la escuela, como en la familia, como un ámbito importante de aprendizaje de las masculinidades. Los datos obtenidos provienen de fuentes secundarias, en especial de la "Investigación sobre los costos para la sociedad, el sistema y las personas acreedoras de las pensiones alimenticias en Centroamérica" realizado por la Fundación Justicia y Género en esta materia, y datos sobre esta problemática en Ecuador. Se destaca la necesidad de transformación en los abordajes de las políticas educativas, la visibilización de factores culturales que impactan en las masculinidades hegemónicas y la paternidad.
\end{abstract}

Descriptores: Derechos humanos; Perspectiva de género; Violencia de género; Masculinidades; Educación.

The hereby article critically investigates the legal institution of alimony and its impact on the economy of women, which constitutes a sort of "Patrimonial Violence" as contemplated in the Inter-American Convention to Prevent, Punish and Eradicate Violence Against Women. The need to incorporate the economic analysis of law is raised, within which the legal phenomenon addresses the need to examine the legal components, but also the value chains from a gender perspective. The sociocultural patterns that deepen and naturalize irresponsible parenthood and its consequences in childhood and adolescence are addressed in relation to the decrease of economic resources to cover the education of their offspring and the absence of coeducation, both in school and in the family, as an important area of learning about masculinities. The data obtained comes from secondary sources, especially from the "Research on the costs for society, the system and people who are entitled to alimony in Central America" carried out by the Justice and Gender Foundation on this matter, and data on this problem in Ecuador. The need for transformation in educational policy approaches is highlighted, as is the visibility of cultural factors that impact hegemonic masculinities and fatherhood.

Keywords: Human rights; Gender perspective; Gender violence; Masculinities; Education.

*Contacto: roxana.arroyo@iaen.edu.ec.

Recibido: 


\section{Alcance y ramificaciones de la problemática del otorgamiento de pensiones alimenticias}

El no pago de las pensiones ciertamente representa una violación a los derechos de la niñez, pero constituye también una forma de violencia contra las mujeres, la violencia patrimonial, y el reforzamiento de las masculinidades hegemónicas. Esta forma de violencia ha sido naturalizada por la sociedad pues se acepta sin cuestionamientos que son las mujeres las que cuidan a su prole. El tema tiene un efecto en la afirmación de los patrones socioculturales, especialmente en sociedades patriarcales como las latinoamericanas.

Es indudable el impacto que tiene el otorgamiento de las pensiones alimenticias y cómo el Estado maneja esta temática dentro de sus políticas de derechos de la mujer y la familia. Se requiere calcular el costo no sólo económico sino vivencial que esto representa para las mujeres, al ser principalmente las receptoras de dicho sustento. La falta de pago de las pensiones afecta no solo a la niñez, también se constituye en un reforzamiento de las paternidades irresponsables, representa una violencia contra las mujeres quienes, además de verse en la necesidad de cubrir los gastos tangibles del cuidado de su descendencia, deben invertir tiempo de sus vidas en la tarea de la crianza, una tarea no remunerada, pero sin la cual sería imposible la continuidad de la especie humana.

Este trabajo incluye referencias a la necesidad de reflexionar sobre los análisis y los aportes de los estudios de la economía feminista, así como la posible inclusión del tema de pensiones en el derecho y la economía, según la cual existe un costo personal que las mujeres asumen, lo que deriva en consecuencias como la imposibilidad de culminar sus carreras o no conseguir ascensos laborales, la brecha salarial entre hombres y mujeres y la falta de recursos para lograr que sus hijos e hijas vean cubiertas el cien por ciento de sus necesidades.

De igual manera, la falta de pago de las pensiones y la ausencia de una paternidad compartida equitativamente entre hombres y mujeres, impacta las posibilidades reales de que los y las hijas puedan acceder a la educación, pues esto recae en las espaldas de las mujeres, que en muchas ocasiones encuentran serias limitaciones por razones de clase, etnia, condición social, migratoria, entre otras, para facilitar el acceso y el acompañamiento a su prole. Este particular permite reflexionar sobre la necesidad de cambios en el aprendizaje social de la paternidad, encaminados a enseñar que ésta es una decisión individual que va más allá del vínculo con la persona que se elige, y de una posible reafirmación de la masculinidad, sino que, es un compromiso personal de vida y no una obligación delegable a las mujeres (Lagarde, 2005, p. 477).

Es evidente que se requieren nuevos paradigmas de aprendizaje sobre la dimensión de la institución de la paternidad; resulta en este sentido un factor importante que, en los procesos educativos, tanto formales y no formales, se incluya la reflexión y el debate sobre la necesidad de una educación no sexista e inclusiva, que sea asumida por los Estados en sus mallas curriculares. 


\section{El difícil camino de la regulación de las pensiones: Un cuestionamiento a la sociedad patriarcal}

La pensión alimenticia/alimentaria ${ }^{1}$ es el derecho de cualquiera de los cónyuges a recibir, por parte del otro cónyuge, dinero o especies para sufragar las necesidades primordiales. Cuando un matrimonio o una unión de hecho se desintegran, la persona que tiene bajo su cuidado sus hijos/as tiene el derecho a acudir ante un juez/a de lo familiar para exigir el pago de los alimentos al padre, la madre o los ascendientes.

La pensión alimenticia/alimentaria incluye la comida, el vestido, la habitación, la educación y la asistencia en caso de enfermedad, entre otros, según sea entendido por las diferentes legislaciones de los Estados (Cillero, 2007). El desarrollo de los derechos humanos y la importancia de incorporar la perspectiva de género en el análisis de la realidad y en el fenómeno jurídico (Facio, 1992), nos lleva a comprender que las diferentes situaciones que se presentan en la sociedad no se dan en el vacío sino en contextos marcados por las asimetrías de género.

En este sentido, la historia del derecho de familia y sus diferentes instituciones no está exenta de esta realidad, la ausencia de una normativa interna de los Estados, especializada en este tema, permitió que fuera regulada en el ámbito del derecho civil con conceptos y normas que favorecían en su mayoría a los varones. Esto nos permite reflexionar acerca de cómo lo referente a las relaciones conyugales, constituye un reflejo de las concepciones estereotipadas sobre el papel de los hombres, las mujeres y la niñez en un sistema patriarcal, de lo que resulta la negativa del reconocimiento pleno de mujeres y niños/as como sujetos de derechos en el seno de los núcleos familiares.

$\mathrm{El}$ tema de las pensiones alimenticias no ha conseguido tener la importancia que merece dentro de las políticas públicas estatales, principalmente del sector judicial, ya que se considera que con las leyes actuales y cambios que son muy paulatinos es suficiente, pero el asunto de fondo es que, con la aparente neutralidad de las normas creadas para regular el tema y, por lo tanto el supuesto que pueden beneficiar de igual manera a los diferentes sujetos involucrados en el cobro de pensiones sean estos hombres, mujeres, la niñez, adultos mayores, personas con discapacidades, entre otros, se obvia un fenómeno que es indiscutible: que las personas más afectadas son las mujeres y la niñez, registrando un alto número de hombres que no asumen su función de cuido, lo que desemboca en el fenómeno de la ausencia de la paternidad responsable como parte constitutiva de las masculinidades.

Son varios los factores que justifican el estudio de las pensiones alimenticias a nivel regional, entre los que se destacan: la afectación a un alto porcentaje de niños/as y adolescentes y su impacto sobre sobre las mujeres y adultos mayores, una legislación que parte de parámetros normativos relativamente similares y problemáticas en los procedimientos, la capacidad de cobertura, los factores culturales, y el costo social que esto implica para las moratorias de pago.

En este punto es importante destacar algunos de los instrumentos internacionales que son fundamentales en esta materia: la Convención sobre la Eliminación de Todas las Formas de Discriminación contra la Mujer (1979), también conocida por sus siglas en inglés

${ }^{1}$ La denominación alimenticia o alimentaria se establece según la legislación interna de los países. 
CEDAW, que obliga al Estado a establecer normas especiales que aseguren una efectiva igualdad ante la ley entre las personas y a eliminar la discriminación contra las mujeres. La Convención Interamericana para Prevenir Sancionar y erradicar la violencia contra las mujeres (1994), también conocida como Belén Do Pará, que prohíbe explícitamente la violencia contra la mujer y consagra su derecho a vivir una vida libre de violencia, la cual viene a complementar la normativa constitucional y a ampliar su interpretación desde una perspectiva de género.

La Convención de los derechos de los Niños (1989), que garantiza los derechos de la niñez y la adolescencia y en donde se fundamenta que son sujetos de derechos, estableciendo principios tales como el interés superior del niños/as (factores que influyen en su crecimiento y desarrollo en condiciones de bienestar), el principio de no discriminación, el derecho a la educación, el derecho a la vida, supervivencia y desarrollo, el derecho a expresar libremente sus opiniones en función de su edad y grado de madurez, así como la responsabilidad del Estado para garantizar los derechos de la niñez. Estas Convenciones se constituyen en un marco jurídico-ético que debe ser tomado en cuenta en la elaboración de políticas a nivel legislativo, ejecutivo y judicial en esta materia.

La disolución conyugal, en el supuesto de unión matrimonial o de hecho, y la fijación de las pensiones de alimentos, son instituciones que forman parte del Derecho de Familia. Este derecho es reflejo del cambio dinámico en las estructuras institucionales de la sociedad que afectaron directamente las relaciones familiares influidas por la cultura, derecho, migración, colonizaciones, técnicas de reproducción asistida, entre otras. La familia como tal se convierte en un "constructo de elementos que convergen en un núcleo humano-afectivo que varía en función del espacio-tiempo; y constituye el pilar básico de las relaciones humanas organizadas" (Ordeñana y Barahona, 2016, p.14).

Resulta relevante recordar que las bases del Derecho Civil Latinoamericano que regulaban esta materia, respondían a una concepción que pretendía consolidar el derecho de los hombres sobre las mujeres y su prole legítimamente reconocida, a esto se remite la indisolubilidad del vínculo matrimonial y la contundente potestad marital que se basaba en el poder del marido y el deber de obediencia de la mujer. En este modelo patriarcal la esposa quedaba subsumida bajo la personalidad legal del marido que implicaba, entre otros aspectos, asumir ya sea el apellido del hombre o su domicilio, careciendo de derechos de propiedad, la imposibilidad de llevar sus negocios propios, entre otras consecuencias (Eicheler, 1999, pp. 447-448).

\section{[...] la institución matrimonial monopolizó la familia, la intimidad y privacidad de las personas; así como sus relaciones sentimentales familiares internas y externas. El matrimonio ha sido una institución ideal para determinar el sistema de producción social, designando la posición que ocupa cada persona en el sistema socioeconómico (trabajo-propiedad-herencia). Por lo que, es posible afirmar que el contrato matrimonial reguló sexo, género, hogar, familia, procreación, cuidado y manutención de los niños, labores domésticas y propiedad. (Ordeñana y Barahona, 2016, p. 17)}

Las normas reforzaron el poder de la masculinidad hegemónica (Amorós, 1987), existiendo las que atraviesan y sostienen toda la estructura genérica. El ejercicio del poder ubicó a las mujeres y a los hombres en espacios diferenciados, a los cuales les corresponde una valoración desventajosa para todo lo que representa al género femenino, sin esto no sería posible la sostenibilidad de la violencia y la discriminación sistémica.

Es importante recordar que la masculinidad hegemónica se fundamenta en una estrategia de poder de dominación en sí misma, muchas veces invisible, que se convierte en una 
práctica cotidiana y donde las instituciones sociales juegan un rol preponderante en su reproducción, por ejemplo, el derecho que, a su vez, es asumido por los propios sujetos en procesos de socialización, naturalizando y legitimando estas prácticas.

Esta cultura, reforzada por la norma y las prácticas jurídicas, justifican lo que actualmente conceptualizamos como micro machismos (Bonino, 2004), que son aquellos comportamientos y hábitos de dominación en la vida cotidiana y en las relaciones. Muchas de estas prácticas son inconscientes y están reforzadas por la naturalización de la aceptación social. Estas pueden ser la no corresponsabilidad y coeducación en el cuidado y educación de los hijos e hijas, que desembocan en la paternidad irresponsable y perpetúan el perverso ciclo de la norma y la cultura.

La evolución de este derecho en América Latina se puede ubicar en la segunda mitad del presente siglo (Huaita, 1999), cuyos cambios se deben en especial a la adopción del principio de igualdad y no discriminación entre el varón y la mujer, que llevaron a la transformación y revisión de los cuerpos normativos con relación a los deberes y derechos de los cónyuges durante el matrimonio o unión de hecho y con posterioridad al mismo, a lo que se sumó la adopción de sistemas de divorcio.

A la luz de estas apreciaciones, es indudable que el marco ético jurídico de los derechos humanos y la expansión del principio de igualdad y no discriminación, a través de la legislación antidiscriminatoria, es entendida como aquellas normas de protección para personas, colectivos y pueblos que se encuentran en condiciones de desventaja, expresadas en exclusiones o limitaciones para el acceso y el ejercicio de los derechos humanos.

Asimismo, es relevante el reconocimiento de los derechos sexuales y reproductivos que, en menoscabo de los derechos de las mujeres, transforman la concepción tradicional que reducía al matrimonio o a la unión de hecho a una única función: la reproducción. Es así como el derecho de familia se regirá por el principio de igualdad de los cónyuges y se asignarán las mismas funciones económicas que serán consideradas de su propio bienestar, así como el de su cónyuge y su descendencia.

En teoría, estos avances en la legislación permitirían que las mujeres ya no sean conceptualizadas como dependientes, sino como iguales a los varones y la prole sea dependiente de ambos, al tiempo que se asuma que padre y madre son responsables del cuidado de su descendencia. Lamentablemente, e incluso en los albores de la segunda década del siglo XXI, la realidad de las mujeres es otra, y este tipo de inequidades no han ido sino en aumento en una era marcada por la diferencia de clase y la predominancia del poder adquisitivo. Asimismo, es claro que los "matrimonios" no se constituyen como única unidad básica de una familia, ya que las uniones de hecho tienen la misma función y por supuesto ya no se admite la diferencia entre descendencia legítima e ilegítima.

Aun así, es evidente que la discriminación y la violencia siguen estando presentes y son el principal obstáculo para lograr la construcción de un paradigma social fundamentado en la igualdad, en el respeto a la diversidad, los derechos humanos y la ciudadanía plena para las mujeres, niñas/os y adolescentes. Desde esta óptica de violencia patrimonial, la división sexual del trabajo, la asignación de las labores de cuidado y crianza como exclusividad de las mujeres, reforzamiento de las masculinidades hegemónicas y la explotación laboral, se consideran todas expresiones diferentes de la opresión, es decir, de esas formas conexas de discriminación en contra de las mujeres y que impactan a sus hijos e hijas. 
Todas estas manifestaciones fortalecen la violencia simbólica como lo establece Bourdieu (1999), se recrean las masculinidades hegemónicas que permiten las paternidades ausentes e irresponsables, las mismas que se refuerzan por la inoperancia del derecho en el cobro de pensiones, instaurándose verdaderos pactos entre los iguales como lo destaca Amorós (1990), y se instituye la naturalización y legitimación de estas conductas vinculadas con la idea de que los hombres, en relación a sus hijos/as, dependen de que vivan con él, obviando que el padre o la madre que no comparten un hogar con su prole, aún tienen responsabilidades económicas con ella.

La preminencia universalmente reconocida a los hombres, se afirma en la objetividad de las estructuras sociales y de las actividades productivas y reproductivas, y se basa en una división sexual del trabajo de producción y reproducción biológico y social que confiere al hombre la mejor parte, así como esquemas inmanentes a todos los hábitos. Dichos esquemas, construidos por unas condiciones semejantes, y por tanto objetivamente acordadas, funcionan como matrices de las percepciones-de los pensamientos y de las acciones de todos los miembros de la sociedad-, trascendentales históricas que, al ser universalmente compartidas, se imponen a cualquier agente como trascendentes (Bourdieu, 1999, p. 49).

Frente a esta cultura en esencia patriarcal, las mujeres se han organizado para reivindicar su condición de humanas y, posteriormente, los derechos de la niñez, cuestionando profundamente los discursos dominantes sobre el papel de lo femenino en el mundo, deconstruyendo los diversos dispositivos de poder y, en el ámbito socio jurídico, cuestionando el concepto de igualdad construido a partir del modelo masculino como paradigma de lo humano, donde hechos que parecieran neutrales como la asignación de pensiones alimenticias para las mujeres y sus hijos e hijas y separaciones conyugales o de uniones de hecho, se convierten en verdaderos campos de disputa por las existentes asimetrías de género (Arroyo, 2007).

La socialización patriarcal (Sánchez, 1988), desde el punto de vista de la autora, considera que el sexo, en cuanto hecho natural, se transforma en hecho social y es parte fundamental de lo que se denomina sistema sexo-género, concepto teórico creado por las feministas anglófonas occidentales de los años setenta y definido por Gayle Rubin (1975) como "el sistema de relaciones sociales que transforma la sexualidad biológica en productos de actividad humana y con el cual se satisfacen esas necesidades humanas transformadas".

La socialización, por lo tanto, se traduce en el modelo cultural impregnado de estereotipos que alimentan los comportamientos esperados, los cuales son dicotómicos y producen las asimetrías de género en las relaciones de poder. Este modelo juega un papel fundamental en la profundización de la violencia y la discriminación de género contra las mujeres, las niñas/os y adultos/as mayores, convirtiéndose en uno de sus principales medios de reproducción. En este entorno social la familia, la educación y los medios de comunicación social, canalizan y transmiten desde lo discursivo, lo simbólico y lo corporal, los roles, funciones y estereotipos de género para hombres y mujeres y que en el caso de las féminas se constituyen en obstáculos para su autonomía y, por tanto, para el ejercicio de sus derechos humanos.

A través de este proceso de socialización se internaliza un modelo dado por la ideología patriarcal, modelo que lleva a una dicotomía entre el espacio público y el espacio privado, el primero asignado fundamentalmente al hombre; el papel de la mujer ha sido considerado históricamente como secundario "ciudadanos de segunda clase", concepción que 
necesariamente se refleja en todos los ámbitos de la vida cotidiana de hombres y mujeres, sustentándose por medio de normas, valores, pautas de crianza y mitos y se explícita en forma contundente en el machismo( Sánchez, 1988).

En efecto, la masculinidad o feminidad (Lagarde, 1997), se construyen en intrincados procesos psicosociales y no por razones naturales, biológicas o religiosas, como se tiende a explicar y justificar, para negar su carácter social y la imperiosa necesidad del cambio, es aquí donde las explicaciones reduccionistas son solo mitos tales como que el machismo es responsabilidad de las mujeres porque son ellas las que educan a la niñez, como si esto fuera natural. En este sentido, como lo menciona Liz Kelly (1987), estas narrativas que desvían y justifican estos procesos son lo que no permiten comprender la violencia contra las mujeres como un continuum producto de una estructura social y no de una manifestación parcial de la cultura.

Una de las manifestaciones de la cultura patriarcal es el poder. El ejercicio del poder ubica a las mujeres y a los hombres en espacios diferenciados, a los cuales corresponde una valoración desventajosa para todo lo representa al género femenino. Sin esta socialización no sería posible la reproducción de la violencia y la discriminación sistémica. Este ejercicio se perpetúa, entre otros factores, por la relación que existe entre la división sexual del trabajo y el establecimiento de los ámbitos público: que corresponde a lo productivo, donde rigen leyes sociales, económicas e históricas y el privado o reproductivo: donde no hay leyes sociales ni históricas, sino la fuerza de la naturaleza (Lagarde, 2014).

Esta visión dicotómica de la realidad ha impactado el derecho internacional de los derechos humanos, ya que la misma no opera en forma neutral respecto a las personas, sino que son espacios ocupados por individuos con sexo concreto, o sea, esta dicotomía de público y privado está atravesada por el género. Asimismo, se convierte en una distinción normativa por la distribución de los poderes y, por supuesto, por los derechos protegidos o no en ambas esferas.

En este sentido, estas asimetrías de género se ven claramente en el derecho por la escogencia y el valor que se le dan a los bienes jurídicos protegidos, tales como el trabajo doméstico, la paga a oficios donde se encuentran mayor número de mujeres como la enfermería, la falta de celeridad en la protección de evasión de bienes en el caso de separación conyugal, la fijación de pensiones o la protección para vivir una vida libre de violencia, por mencionar algunos. Saltan a la vista las valoraciones que resultan de estos complicados procesos de socialización, por medio de los cuales las diferentes instituciones han asignado un valor político, jurídico y económico a dichos bienes, es más, deciden cuáles bienes deben ser tutelados o no y cómo. Esta asignación, por supuesto, no es neutral desde una perspectiva de género (Arroyo, 2002).

En esta breve reseña, la evolución del derecho de familia, sus instituciones, sus intrincados procesos de socialización, que son el resultado de la cultura dominante patriarcal, deberían ser aspectos relevantes para la educación inclusiva, entendiendo a esta como la conceptualiza La Organización de las Naciones Unidas para la Educación, la Ciencia y la Cultura (UNESCO, 2005) en tanto que debe responder a la diversidad de las necesidades de todos los estudiantes, planteando la educación como un proceso que facilita y permite la participación en el aprendizaje, las culturas y las comunidades, para así lograr disminuir la exclusión.

Asimismo, es importante que esta propuesta de inclusión este encaminada hacia el logro de una educación no sexista, que plantee contenidos que desmonten la naturalización de 
los roles, funciones y estereotipos de género relacionados con las concepciones de familia y en especial procesos pedagógicos que transmitan nuevos valores como la igualdad, la paternidad responsable, la coeducación, las familias, entre otros. Se requiere enfocarse en contextos diferentes que aporten en el convencimiento de una sociedad no sexista, dos de estos contextos que se consideran fundamentales son la familia y, por supuesto, el escolar (García, 2012, p. 9).

Así pues, entendemos que la educación, necesariamente aplicada desde planteamientos y/o metodologías inclusivas, ha de constituir la base sobre la que construyamos aprendizajes activos, críticos y reflexivos con nuestro alumnado. La labor de los maestros y maestras ha de centrarse en ser capaces de trasladar la importancia de entender las desigualdades que al día de hoy aún persisten en nuestras sociedades en relación al género, y entrenar la mirada de los que se convertirán en los futuros/as ciudadanos/as para ver las desigualdades entre géneros y participar activamente para cambiarlas (Tortonda, Barriga y Gonzáles, 2019, p. 70).

\section{El impacto de la disolución del vínculo conyugal en la economía de género}

Existe una amplia gama de temas relacionados con las asignaciones de género que se han mediatizado a través de diferentes discursos justificativos como: la no valoración del trabajo doméstico no remunerado de las mujeres que ha jugado en su contra para la fijación de los bienes gananciales en los juicios de divorcio, la construcción de la masculinidad que refuerza la paternidad irresponsable al otorgar cierta permisibilidad social cuando se incumplen las obligaciones alimentarias hacia sus hijas e hijos. Una especie de impunidad de facto se genera por el tratamiento que se da a estos derechos en la administración de justicia, cuando son reclamados por las mujeres, niños/as y adolescentes.

La socialización y la reproducción de los estereotipos de género van a jugar un papel fundamental en los roles y percepciones de la sociedad sobre el papel de las mujeres y hombres en casos de regímenes patrimoniales, separación de patrimonios, participación de las ganancias, el derecho alimentario entre ex cónyuges, pensiones alimentarias sobre la prole, entre otros. Es claro que se trata de una feminización de la pobreza de las mujeres, dado el tratamiento que existe en muchas legislaciones o por los criterios aplicados en el tema de pensiones alimentarias y separación que afecta su patrimonio.

Es aquí donde el acceso a la justicia desde una perspectiva de género es de fundamental importancia, tanto para la valoración de los derechos de las mujeres, los derechos de la niñez y adolescencia, como de las personas adultas mayores. Cabe mencionar algunos instrumentos relevantes al respecto: tenemos el caso de las Recomendaciones Generales del Comité de la CEDAW No 19 (1992), la Recomendación General número 28 (2010), relativa a las obligaciones básicas de los Estados partes de conformidad con el artículo 2 de la Convención sobre la eliminación de todas las formas de discriminación contra la mujer, Recomendación General 25 (2004) 25 sobre el párrafo 1 del artículo 4 de la Convención sobre la eliminación de todas las formas de discriminación contra la mujer, referente a medidas especiales de carácter temporal, Recomendación General 33 (2015) sobre el acceso a la justicia a las mujeres y del Comité de la Convención de los Derechos del Niño, especialmente la Observación general $\mathrm{N}^{\circ}$ 14 (2013) sobre el derecho del niño a que su interés superior sea una consideración primordial (artículo 3, párrafo 1), y las 
diferentes ponderaciones que se deben tener en cuenta en los derechos de las mujeres y el interés superior de los niños, el principio de igualdad y la posible interseccionalidades de discriminaciones que se dan en las diferentes negociaciones.

Estos estereotipos, a pesar del avance en el Derecho de Familia, impactan no sólo en algunas normas, sino en el tratamiento que la administración de justicia da a estos casos. Es así como los principios de la concepción de la familia -patriarcal heterosexual- siguen existiendo en el imaginario y en la realidad, y entran en contradicción con los principios de una visión de la modernidad sobre las familias y su regulación, dejando de lado a las familias extensas, monoparentales, transfronterizas y transnacionales (Pedone, 2010), así como a las pertenecientes a la diversidad sexual (Corte-IDH-, 2017).

No se debe perder vista lo que la Recomendación General No 28 nos indica: que la discriminación puede ser dirigida contra la mujer por razón de su sexo y género. El género se refiere a la construcción social de identidades, atributos y roles de mujeres y hombres y el significado cultural impuesto por la sociedad a las diferencias biológicas, que con frecuencia se reproducen en el sistema de justicia y sus instituciones (RCG 28: Numeral 2). Asimismo, en relación con esto, en virtud del artículo 5.a) de la CEDAW, los Estados Partes tienen la obligación de detectar y eliminar las barreras sociales y culturales subyacentes, incluyendo los estereotipos de género, que impiden a las mujeres ejercer y reclamar sus derechos y menoscaban su acceso a recursos eficaces.

Los estereotipos comprometen la imparcialidad y la integridad del sistema de justicia, están presentes en todas las fases de los procesos e influyen en la sentencia y resoluciones, lo que va a afectar directamente la situación económica de la mujer colocándola en situaciones de riesgo y vulnerabilidad. Es necesario recordar que las mujeres, los niños, niñas y adolescentes deben poder confiar en un sistema de justicia libre de mitos y estereotipos, y en un poder judicial cuya imparcialidad no se vea comprometida por estas suposiciones sesgadas. La eliminación de los estereotipos en el sistema de justicia es un paso crucial para garantizar la igualdad y la justicia para las acreedoras de los derechos enunciados, en especial las pensiones alimenticias.

Desde este punto de vista existe una vinculación entre los estereotipos y las relaciones de poder generadas por las asimetrías de género que resultan en múltiples formas de discriminación, es importante en este análisis señalar que el fundamento de la discriminación son los prejuicios que fundamentan estos estereotipos presentes en los entramados de la cultura que refuerzan las diversas formas de opresión existentes en nuestras sociedades.

El poder, al ser un sistema de relaciones, implanta en el espacio de los iguales una red de fuerzas constituidas por quienes ejercen el poder y se reconocen a sí mismos como sus titulares legítimos, teniendo en cuenta que, junto a ellos, existe un conjunto de posibles titulares que guardan su turno ante la posibilidad de un relevo. Los iguales existen en tanto tienen algo que repartirse: su dominio y hegemonía sobre las mujeres (Cobo, 1995, p. 36).

En este escenario, las mujeres han sido históricamente las encargadas del espacio reproductivo y, si bien han incursionado y su presencia actualmente es mayor en lo público, el cambio dirigido a democratizar el ámbito privado es lento en la consecución de lograr la conciliación del mundo laboral y la vida personal y familiar (Satzman, 1989), así como la comprensión de que la realización de los trabajos domésticos y de cuidados es también responsabilidad masculina, como resultado de procesos de reeducación de los 
hombres. Es en este transitar consciente, donde los hombres desarrollan recursos encaminados al fortalecimiento de personalidades, que estén en mayor concordancia con modos de vida más democráticos y paternidades responsables (Lagarde, 2014).

En el análisis del Derecho de Familia es importante comprender que el poder está presente en las diferentes circunstancias y que los sujetos tienen posiciones distintas, por lo tanto, no se puede obviar que el patriarcado se reproduce constantemente al interior de las familias, logrando fortalecer culturalmente la asimilación del género como un elemento dialéctico de las construcciones sociales de las identidades, funciones de atributos para los varones y las mujeres y por supuesto de la prole que se relaciona con ellos/as.

Por todo lo expuesto es importante comprender que el derecho, como instrumento que regula las relaciones sociales, reproduce en muchas formas la subordinación de lo femenino y el modelo de familia en lo que es la consciencia colectiva y personal de nuestra cultura, y no deja de jugar un papel preponderante todas las instituciones que refuerzan esto, como lo son: el derecho, la economía, las ciencias, las religiones, los medios de comunicación, entre otros. En este sentido, es importantísimo repensar el derecho y ver cuáles son las nuevas prácticas que consoliden nuevos paradigmas que permitan avanzar hacia la igualdad y no discriminación en la aplicación de las instituciones jurídicas tales como las pensiones alimenticias y la disolución del vínculo conyugal.

Si bien existe todo un desarrollo en la línea de los estudios socio legales que señalan que la relación entre las normas y las prácticas cotidianas no es tan directa, también es cierto que las introducciones de nuevos valores que conforman derechos como "el derecho a vivir una vida libre de violencia" impactan a mediano plazo los patrones socioculturales existentes, por supuesto que no es la norma en sí misma, sino un conjunto de factores que lo facilitan, tales como las políticas públicas, los movimientos sociales, la educación, por mencionar algunos.

En relación al punto anterior, resulta de mucha utilidad el método del análisis jurídico propuesto por la jurista Alda Facio (1992), que nos propone se debe estudiar el componente formal normativo donde encontramos las leyes que serían sinónimos de los que muchos/as tratadistas llaman la norma agendi, es decir la ley formalmente promulgada o al menos formalmente generada, ya sea como la ley constitucional, tratado internacional, leyes sustantivas y adjetivas, decretos, reglamentos, convenciones colectivas, entre otras. Aquí vamos a encontrar las normas ya referidas como la Convención de los Derechos de los niños ratificada por Ecuador en 1990, así como la Constitución Política (2008) Código de la Niñez y Adolescencia (CONA) 2003, entre otras leyes conexas.

El componente estructural, entendido como la interpretación que se hace del componente normativo, es formulada tomando en cuenta lo que dice la norma, o creada por la administración de justicia a través de sus sentencias y, en muchos casos, por normas que han sido derogadas, pero continúan en la mente de los / as operadoras de justicia, la policía, los administrativos, los que otorgan servicios estatales tales como en el ámbito de la migración, la educación, la salud, etc.

En cuanto a las personas funcionarias que realizan la tarea de seleccionar, combinar, aplicar e interpretar, lo importante de destacar de esta función es la posibilidad de favorecer o no los avances normativos, debido a que si prevalecen en ellos/as más sus visiones vinculadas a roles estereotipados de género, por ejemplo en el tema de la violación, el estereotipo de la víctima provocadora, se buscará culpar a las mujeres 
preguntando sobre su comportamiento moral y sobre como vestía, por qué había salido sola a esas horas de su casa, etc., desvirtuando la norma que busca la protección al derecho de la libertad sexual y la integridad de las víctimas.

En cuanto al componente político-cultural, está vinculado a las cotidianidades de la gente, donde se ubican las reglas no escritas, no formalmente promulgadas o ya no vigentes, pero que aún prevalecen en el imaginario, es ahí donde las costumbres, actitudes, tradiciones y conocimiento que la gente tiene de la ley, así como el uso que la gente haga de las leyes existentes, de las que en la vida diaria, siguen vigentes aunque hayan sido derogadas, así como de las relaciones entre las leyes no escritas y las escritas. O sea, que también en este componente existen normas no escritas, aquellas que no están formalmente promulgadas pero que, además de ser obedecidas por la mayoría, son formalmente reforzadas. Por ejemplo, en el caso de la violencia domestica/intrafamiliar, aún prevalece en las relaciones sociales la naturalización de la violencia psicológica, física y sexual en los vínculos afectivos, aunque exista la Convención Interamericana para Prevenir, Sancionar y Erradicar la Violencia contra la Mujer de 1994. Estos tres componentes están relacionados entre sí de tal manera que se influyen, limitan y/o definen el uno al otro a tal grado, que no se puede conocer el contenido y efectos que pueda tener una determinada ley, un principio legal o una doctrina jurídica, si no se toman en cuenta en su conjunto.

Existen serias limitaciones para obtener información detallada, pertinente, precisa y actualizada en la región. Además, persiste la heterogeneidad metodológica que dificulta la comparabilidad entre indicadores. Esta situación, más allá de dificultar el estudio y el acercamiento a esta problemática sobre los orígenes y las profundidades de la desigualdad sexual, implican muchas áreas de mejora respecto de los objetivos de transparencia en las instituciones del Estado.

Por otra parte, aunque se dificulta un análisis sobre la direccionalidad del impacto -es decir, si la pobreza de las mujeres impacta su situación en lo relativo a los derechos patrimoniales, o si es el sistema judicial el que impacta en la pobreza de las mujeres- cabe preguntarse si un examen estadístico más exhaustivo resultaría insuficiente, si no se tiene claro que las estructuras de los sistemas judiciales, cuyas transferencias de valor reflejan resultados con costos de una demanda (por pensiones o división de bienes gananciales), considerablemente mayores para las mujeres, perpetúan y reproducen las disparidades entre sexos presentes en la región, en términos agregados, en lugar de compensarlas.

En este sentido, para dar respuesta a la pregunta anterior, creemos adecuado el uso de cadenas de valor que describe el conjunto de actividades requeridas para llevar un producto o servicio desde que éste es concebido, pasando por diferentes fases de producción hasta la entrega al consumidor final y su disposición después de su uso (Flores \& Lindo, 2005, p. 12) y utilizado por los estudios feministas en esta materia, entendiendo este concepto como:

El estudio de cadenas de valor constituye una de las herramientas, heredadas de la
economía tradicional, de las que se sirve la Economía Feminista para explicar los
mecanismos estructurales de desigualdad sexual presentes en las dinámicas del
mercado capitalista y materializadas en las facetas de la demanda agregada
(Jiménez, 2019, p. 31)

En esta línea, se puede hablar de una prevalencia en la violencia patrimonial que se transfiere desde la estructura económica y social, por medio de procesos como los que propone el tema de las pensiones, dirigida hacia las mujeres usuarias. Al aplicar el método del análisis económico del derecho, se podría abordar con mayor precisión las cadenas de 
valor, en pensiones alimenticias, y los procesos por separación de bienes gananciales, logrando calcular los costos de estos procesos, los cuales impactan en mayor medida a las mujeres en términos de bienestar, y las transferencias que ocurren sobre la base de la desigualdad de ingresos y de trabajos entre hombres y mujeres.

Esto, bajo la lógica de encadenamientos, implica el cálculo de las desmejoras importantes en el bienestar de las demandantes y sus familias. Situación que se reintegrará de manera cíclica en las cadenas de valor, ya que los sujetos involucrados en cada uno de los eslabones tienen posiciones diferentes de poderes de negociación diferenciados entre hombres y mujeres.

Introducir la perspectiva de género implica, por lo tanto, tomar en cuenta otras variables y darle valor, para con ello ampliar el espectro a otras relaciones sociales que intervienen en el mercado y que no suelen visualizarse como, por ejemplo, el cuido, trabajo gratuito de las mujeres, el tiempo invertido, traslados, trámites post fijación de la pensión, entre otros. Y, por supuesto, esto tiene una transferencia de valor en términos de costos, gastos y ganancias entre los eslabones.

Es así que los tramites que se alargan, las múltiples gestiones y traslados, el cuido de los niños y niñas, los permisos que se deben solicitar para impulsar las gestiones en caso de tener empleo formal y en relación de dependencia y, con mayor razón, las mujeres que están en el sector informal, el establecimiento de las pensiones, la carga emocional de los prejuicios todo esto tiene un costo social y que se puede valorar económicamente en donde la demandante lleva un mayor peso y el demandado uno menor. Por lo tanto, no es solo que la norma, aunque esté bien elaborada no se aplica adecuadamente o que la sociedad considere que las mujeres lucran de su prole, sino que esto tiene un costo económico adicional, y se constituye en un problema social a menos que se elaboren procesos mucho más ágiles y eficientes. Por lo que resulta pertinente, por el lado de los procesos de justicia, simplificar trámites y reducir tiempos, en la medida que se brinde un servicio de calidad.

En este sentido, las economías padecen, en términos nominales, de costos asociados a las demandas de familia, el peso de los procesos se traslada en el corto plazo a las mujeres que los interponen. Pero, en el largo plazo, el impacto se asociaría con una desmejora en el bienestar general de las familias que participan de estos, incluso en el bienestar general de la población. Queda planteada la tarea de extender y precisar el alcance de este tipo de análisis económico con enfoque de género para el caso de los procedimientos, tales como las pensiones alimenticias y las divisiones de bienes gananciales, que pretenden buscar una sociedad más justa y equitativa. Como dice la economista Daniela Jiménez (2009) "Debe entenderse que estos procesos sociales se generan en el espacio de la meso-economía, son conectores entre lo micro y lo macro, y es por ello por lo que podemos ver los efectos como impactos para las economías nacionales, la región, las poblaciones y los agentes en particular" (p. 55).

\section{La situación de las pensiones alimenticias en Ecuador}

En Ecuador se ha asumido esta problemática en diferentes momentos, en una línea del tiempo sobre esto es importante partir del fenómeno de la dolarización que se da en el año 1999, si tenemos presente que el cálculo inicial del cambio de sucres a dólares estaba estimado en un dólar por 25000 sucres y el promedio de las pensiones era de 1000 sucres por pensión, esto significó un equivalente que oscilaba entre los 4 a 5 dólares; el Estado, 
con el fin de dar respuesta a esta situación y otras que ya acarreaba en relación a las pensiones, emprendió una sería de cambios para limitar la discrecionalidad de parte de las /os operadores de justicia. Es así como el 28 de julio de 2009 se publicó la Ley Reformatoria del Título V del Libro II del Código de la Niñez y Adolescencia, remplazando el anterior código en lo referente a los alimentos, la forma de calcularlos y el procedimiento para demandarlos. Para limitar la discrecionalidad de los operadores/as de justicia, se aprueban las tablas de fijación mínima de pensiones y sus actualizaciones se establecen según los salarios básicos unificados vigentes.

Es importante destacar que Ecuador fue el primer país en América Latina en ratificar la Convención Sobre los Derechos del Niño de las Naciones Unidas (CSDN) en 1990, esto influyó en la posibilidad de iniciar procesos de revisión y adecuación de la normativa interna para acoplarla a los estándares internacionales. Un primer avance se hace en el año 1992, donde se introducen una serie de reformas al entonces Código de Menores pero que en realidad queda muy corta con relación a los estándares internacionales. No es sino hasta el 2003, con la aprobación del Código de la Niñez y Adolescencia (CONA), que entra en vigor junto con su publicación en el Registro Oficial No. 737 del 3 de enero de 2003, que se puede evidenciar un avance cualitativo en esta materia y en el año 2009 un nuevo cambio muy importante a través de la publicación de la Ley Reformatoria del Título V del Libro II del Código de la Niñez y Adolescencia, encaminada a una modificación en el código a todo lo referente a los alimentos, la forma de calcularlos y el procedimiento para demandarlos, lo que limita la discrecionalidad de las/os operadores/as jurídicos para calcular los mismos (Romo, 2019 ).

En el año 2017 se abre un nuevo debate a nivel de la sociedad donde participan diferentes sectores y asociaciones y se impulsa una reforma sobre aspectos como la fijación de la Tabla de Pensiones Mínimas de Alimentos, con relación a los obligados subsidiarios, las medidas de apremio, entre otros. La reforma al Código de la Niñez y la Adolescencia, que fue presentada por El Ministerio de Inclusión Económica y Social (MIES) y el Consejo Nacional para la Igualdad Intergeneracional (CNII), hace alusión a 136 artículos para ser valorados por la Asamblea Nacional (El Universo, 2020)2. Pese a estos avances, los números de personas que se encuentran en mora, cobro de pensiones a personas que se encuentran en el extranjero, boletas de apremio corporal por falta de pago, y por supuesto el manejo y la presencia de estereotipos que principalmente son dirigidos a las mujeres que son las que solicitan la pensión para su prole, subsisten en la actualidad.

En Centroamérica, a manera de referencia sobre problemáticas similares, los obstáculos que enfrentan las usuarias se ven reflejados claramente en el caso de Panamá, donde la opinión de los/as operadores / as de justicias es que el procedimiento debería ser ágil, pero hay una serie de factores que perjudican la efectiva labor de las y los jueces/ juezas de Despacho; recargo de labores, prolongando los procesos de tres meses a un año, sin tomar en cuenta los tramites de desacato y morosidad (Fuente Investigación sobre los costos para la sociedad, el sistema y las personas acreedoras de las pensiones alimenticias en Centroamérica: Juzgado Primero, Tercero, Cuarto Municipal de Familia del Distrito de Panamá, 2018).

\footnotetext{
2 https://www.eluniverso.com/noticias/2020/01/16/nota/7693893/reforma-codigo-ninezpropone-pension-alimenticia-hijos-hasta-24
} 
Con relación a los prejuicios de las sociedades sobre las pensiones, esto se evidencia en los estereotipos, en este sentido resulta interesante el estudio realizado por la Defensoría Pública de Ecuador en el año 2018 que brinda cifras a destacar:

\begin{abstract}
[...] Evidencia que el 82 por ciento de las pensiones oscila entre los 51 y 200 dólares, y que el 93 por ciento está entre 51 y 300 dólares, mientras que el motivo de la alarma social, sobre pensiones elevadas, representa el 0,0006 por ciento. Es decir, el prejuicio y el desconocimiento posicionaron ante la sociedad ecuatoriana la idea de que las mujeres hacen negocio con las pensiones de alimentos. Lo que no se dijo es que de esas pensiones hay una mora del 69 por ciento, lo que significa que el Ecuador debe hablar, por un lado, de pensiones fijadas, $y$, por otro, de pensiones pagadas. (Pazmiño, 2018, p. 8)
\end{abstract}

Esta situación se corrobora en la información que sobre el tema dan los medios de comunicación cuando lo abordan, y algunos sectores en sus opiniones refuerzan las asignaciones de roles de que son las mujeres las cuidadoras porque naturalmente les corresponde, asimismo, en sus argumentaciones prevalece la estigmatización de que en realidad es una manera de aprovecharse y un estilo de vida el embarazarse para cobrar estas pensiones, pero nadie se refiere a los padres ausentes y la responsabilidad de la planificación y decisión de tener prole o no, pareciera que aún prevalece en nuestras sociedades la idea del varón engañado, el cual no puede contener sus instintos y es un simple espectador en relación a su deseo o no de paternidad.

Sobre este nudo crítico de las representaciones culturales en esta materia, vale la pena destacar el debate sostenido durante el periodo 2018-2019 en Ecuador, por los colectivos, el gobierno y otros sectores, referido a la boleta de apremio corporal como un mecanismo necesario o no y si este resolvía el problema o lo agudizaba en la consiguiente falta de pago.

En las argumentaciones, prevalecieron en parte las opiniones que reproducían los estereotipos, tales como que son las mujeres las que lucran de estas pensiones y que esta se constituye en una venganza de las féminas contra los hombres. Según criterio de Cecilia Espinosa, miembro del Observatorio General del Colegio de Abogados, la pensión sí es proporcional a los ingresos del demandado, tomando en cuenta sus gastos y cargas familiares, y rechaza que las mujeres lucren de este ingreso que no es para ellas sino para sus hijos (Pichincha- comunicaciones) ${ }^{\text {s. }}$

Es innegable que esta situación requiere de otras miradas y preguntas, pues los datos que nos proporciona la Defensoría (Boletín, 2018), nos evidencian una problemática a profundizar y estudiar y que afecta a la población infantil y adolescente y por supuesto a las mujeres, pues son las representantes de los intereses de sus descendientes, sin obviar que nos permite reflexionar sobre el impacto diferenciado para los hombres.

Se requieren procesos de reeducación que permitan reflexionar sobre la importancia de incorporar la paternidad como parte de las masculinidades y que sean producto de sus elecciones, desarrollo emocional, ejercicio consciente de sus derechos sexuales y reproductivos y no como sucede en muchos casos: comprobación de una virilidad.

El estudio realizado por la Defensoría, nos brinda datos que nos permiten dimensionar los alcances del problema y las necesidades de la niñez y las mujeres. El gráfico No 1 describe

\footnotetext{
$3 \quad$ http://www.pichinchacomunicaciones.com.ec/expertos-destapan-problemas-de-pensiones-yregimen-alimenticio/
} 
los procesos y cuáles son las materias requeridas, observamos que el tema de alimentos es el problema más relevante con 49.409 casos. Otros ítem están íntimamente vinculados, tales como la liquidación de alimentos, alimentos internacionales, alimentos de mujeres embarazadas, alimentos voluntarios y temas conexos que tienen relación con la pretensión de pensiones, por ejemplo, reconocimiento de paternidad; estos números revelan la magnitud del problema en temas de tramitación y expectativas de las mujeres peticionarias, según los datos de la Defensoría.

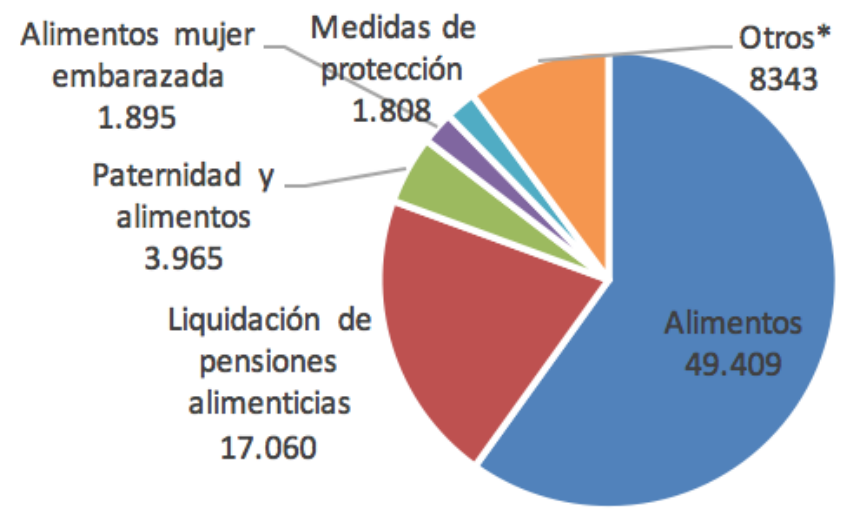

Figura 1. Solicitudes ciudadanas de atención en familia y alimentos por temas de atención

Fuente: Elaboración propia.

En relación con lo anterior, la figura 2 se refuerza de forma muy clara cuáles son las prioridades y las expectativas de las usuarias para poder responder a las necesidades de su prole, los temas principales son: incidente de rebaja de pensión de alimentos, medidas de protección, alimentos para mujer embarazada, paternidad y alimentos, liquidación de pensiones alimenticias y alimentos con un 59,90\% de atención.

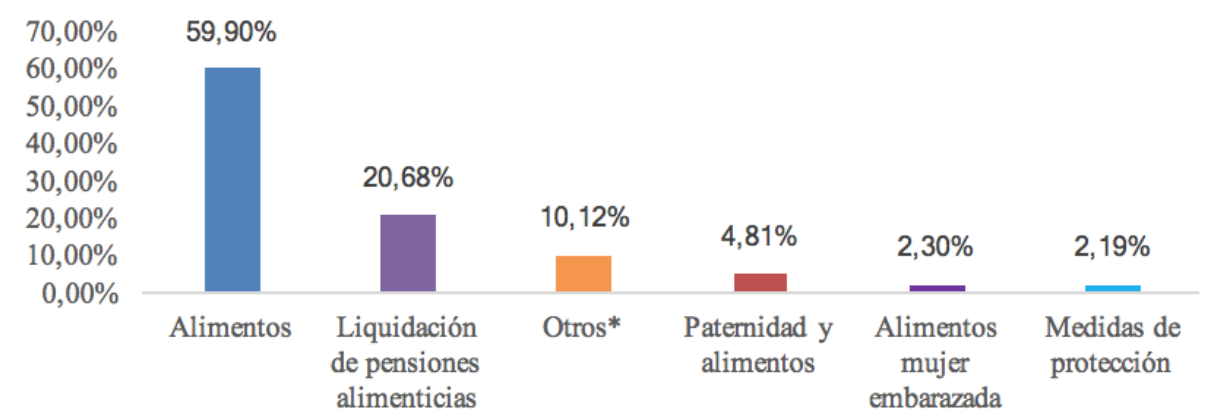

Figura 2. Porcentaje de los principales temas de atención en familia y alimentos, 2018 Fuente: Sistema de Gestión de Información de la Defensoría Pública. Elaborado por la Dirección Nacional de Investigaciones Aplicadas. Elaborado por Michelle Aguirre.

Esta tendencia se mantiene igualmente en el período enero-diciembre del 2019, como lo demuestran los siguientes datos de la Defensoría ( boletín 2019) en lo que se clasifica como los 10 principales temas en el patrocinio en materia no penal, es así como en el primer lugar se ubica la materia de alimentos 56.648, seguido por liquidación de alimentos 21.473 y paternidad y alimentos4.992, y relacionado con esto en el puesto quinto encontramos 
alimentos de mujeres embarazadas 2131 y en el séptimo y noveno respectivamente se refiere a incidentes de rebajas de pensiones alimenticias 1534, incidentes de aumento de pensiones 1395 .

En un estudio realizado por el Ministerio de Justicia, Derechos Humanos y Cultos de Ecuador en el año 2011 y que se llevó a cabo en los Juzgados de la Niñez y de la Adolescencia, se destacaba que una de las consecuencias de las rupturas de las relaciones de las mujeres está vinculada al empobrecimiento de las mismas, ya que estas se hacen cargo de la prole y que en un alto porcentaje deben asumir solas el cuidado y la manutención de los hijos/as, lo cual constituye un proceso revictimizante.

En este mismo sentido, la investigación realizada en Centroamérica nos permite evidenciar que, es fundamental evitar en los procesos resultados revictimizantes, los cuales se dan porque las usuarias suelen pasar por diferentes personas: recepcionista, abogado/a, la persona trabajadora social, los/as psicólogas/os, peritajes, etc. Además, la aportación de documentación puede llevar meses, inclusive en la misma audiencia puede tener que repetir el relato a la jueza/es, esto tiene un costo emocional y económico muy alto, si se toman en cuenta los permisos en su trabajo que en muchos casos implica un descuento de su salario ( Fuente: Grupo de la Investigación sobre los costos para la sociedad, el sistema y las personas acreedoras de las pensiones alimenticias en Centroamérica: grupo focal juezas y jueces de Familia provenientes del municipio de San Salvador, 2018).

En la misma línea, la investigación del Ministerio de Justicia, Derechos Humanos y Cultos de Ecuador destaca que la fijación del monto mínimo que se obtiene para la pensión está fuertemente ligada a la condición de género y la interseccionalidad de variables como clase, etnia, condición social, entre otras, que se vuelven verdaderos obstáculos para el acceso a la justicia, impactando aún más a aquellas mujeres de menos recursos económicos y culturales, sin tomar en cuenta factores de orden simbólico y cultural, que ubican a las mujeres en el "deber ser" de entrega absoluta cuando carecen de preparación para llevar adelante sus causas.

[...la mayor parte de las mujeres que apelan al derecho de sus hijas e hijos por pensión de alimentos son "víctimas" de la violencia económica, pues luego de la separación se ven empobrecidas de manera dramática y no tienen cómo solventar la manutención de la prole. Muchas quedaron con el monto provisional [...] y tienen a su cargo todo el trabajo doméstico y cuidado de sus hijos/as, tareas que no han sido contabilizadas como aporte económico de las mujeres (Ministerio de Justicia, Derechos Humanos y Cultos: Projusticia; Observatorio de los Derechos de las Mujeres, Acuerdo por una Justicia, CEPLAES, 2011 p. 30).

Según este estudio, los procesos duran meses y tienen un costo alto, sumado al hecho de que los operadores/as de justicia carecen de una perspectiva de género. Esto se agrava si tomamos en cuenta el traslado, las copias, y muchas veces el tener que localizar al demandado, la presentación de pruebas y la interminable situación de pensiones impagas y la readecuación de las mismas, así como la falta de conocimiento de las usuarias.

En esta misma problemática existe cierta relación con los resultados obtenidos en Centroamérica, en cuanto a que las usuarias de estos servicios no conocen sus derechos o los conocen parcialmente, y llegan con mucha desorientación en cuanto a la normativa y sus posibilidades y en muchos casos con miedo, lo que incide en no tener una clara estrategia. Esto va más allá de que la normativa y los procedimientos sean sencillos, es aquí donde el derecho no toma el contexto de una sociedad sexista y pretende que existe 
un espacio neutral de género (Investigación sobre los costos para la sociedad, el sistema y las personas acreedoras de las pensiones alimenticias en Centroamérica 2018-2019).

Además, si se aplica el análisis económico del derecho, podemos enfatizar que es necesario calcular en las cadenas de valores los costos para la persona demandante, costos para el obligado y costos del Estado, de esto se desprendería que las mujeres incurren en gastos más elevados independientemente del país o proceso, esto por el impacto de elementos como las horas de trabajo no remunerado y los días de movilidad, y que estos gastos impactan en mayor medida a las mujeres en términos de bienestar, puesto que las transferencias ocurren sobre la base de la desigualdad de ingresos y de trabajos entre hombres y mujeres (íbid p. 17).

\section{Conclusiones}

Es necesario introducir en las políticas y fundamentos de la educación, nuevos paradigmas de contenidos, aprendizajes y procesos pedagógicos, tanto en la educación primaria, secundaria y de las instituciones superiores, encaminadas al cambio de estos patrones socioculturales que profundizan las concepciones sobre la institución de la paternidad. Se requiere introducir y analizar problemáticas como la descrita sobre pensiones, su impacto en la vida de mujeres y la niñez y su vinculación con las masculinidades hegemónicas, como un problema que debe ser abordado desde la academia.

La trasformación de los patrones socioculturales que profundizan la violencia y la discriminación basada en estereotipos está íntimamente vinculada con la construcción de nuevos paradigmas que respondan a una educación no sexista e inclusiva, la cual integre la perspectiva de género y de derechos humanos, lo que permitirá plantear el análisis del sistema patriarcal y sus nefastas consecuencias en la construcción de las sociedades.

Se hace imperativo comprender que el tema de las pensiones es un factor que afecta el derecho a la educación de niños, niñas y adolescentes, ya que éstos dependen en su totalidad de los ingresos de sus madres las cuales, en un porcentaje alto por sus condiciones de género, clase, etnia, entre otras, enfrentan interseccionalidad de discriminaciones que afectan directamente las posibilidades reales de responder a los requerimientos del acceso a la educación de sus hijos e hijas.

Es así como, a pesar de que en el componente formal-normativo, como se plantea en la metodología del análisis del fenómeno jurídico explicado anteriormente, se constata un avance en esta materia en lo que se refiere a las normas, esto no es suficiente, pues en relación al componente estructural se evidencian falencias, ya que persisten los obstáculos que van vinculados a la interpretación de los/as funcionarios/as de la administración de justicia en esta materia, y no es suficiente contar con principios que rijan los procedimientos de pensiones si estos resultan ser engorrosos y poco ágiles.

Con respecto al componente político cultural, sigue presente en el imaginario la sociedad el hecho de que las mujeres deben hacerse cargo de su prole, y si sucede que protestan o reclaman el derecho a pensión de sus hijos/as, se enfrentan a la inercia del sistema, son estigmatizadas e inclusive se las acusa de querer lucrar. Esta naturalización opera en sentido contrario en muchos casos para los hombres que no pagan las pensiones, evaden los procedimientos o consideran que eso les corresponde a las mujeres, afectando por supuesto los derechos de su prole. 
Existe una impunidad que afecta desproporcionalmente y que, si bien afecta directamente a la prole, su consecuencia también es el empobrecimiento del patrimonio de las mujeres, constituyéndose en una violencia patrimonial. Con relación a los hombres, refuerza las masculinidades pues su construcción la paternidad, en muchos de los casos, está muy lejana de ser parte de su construcción identitaria.

Es importante entender que los procesos jurídicos de las pensiones van más allá de obtener un beneficio económico para las necesidades de la niñez y los adolescencia, sino que trascienden en el impacto de la cobertura de necesidades para las poblaciones que, en su mayoría, son mujeres según las estadísticas existentes, y que estas cifras nos revelan el accionar del sistema patriarcal en nuestras culturas y sus intricados procesos de socialización, los cuales llevan a la naturalización de roles y funciones que les asigna el mandato del cuido casi exclusivamente a las mujeres y refuerza las masculinidades hegemónicas, en lo que respecta a la paternidad y su significado.

Asimismo, es claro que el derecho no se da en contextos neutrales, la interseccionalidad de discriminaciones está presente en la violencia patrimonial por falta del pago efectivo de las pensiones alimenticias, impacta a la parte de la población que tiene menos acceso al trabajo remunerado y que está sobre-representada en los indicadores de pobreza, la cual además sufre de violencia estructural, la misma que se manifiesta en el acceso a recursos productivos y financieros, y que son objeto de la naturalización histórica de la violencia de género sexista y de altos niveles de explotación laboral en el hogar, ya que son las mujeres quienes destinan más tiempo a realizar trabajos no remunerados.

El tema de las pensiones alimenticias es una deuda pendiente por parte de los Estados, es claro que se requiere de mayores estudios que permitan dilucidar las dimensiones del problema y los obstáculos que surgen para el acceso a la justicia en esta materia, que van desde las normas, los mecanismos, las interpretaciones que hacen los /as operadores de justicia, hasta las características de los sujetos involucrados.

En este sentido, es importante hacer estudios sobre impacto de la dinámica de las pensiones alimenticias desde diversos enfoques disciplinarios, por ejemplo, la conjugación del derecho y el análisis económico del mismo, son métodos y planteamientos teóricos que permiten profundizar el fenómeno jurídico y los posibles costos monetarios para el Estado y los sujetos involucrados en los trámites relacionados con las pensiones alimenticias, que no cumplen con los principios de celeridad y eficacia necesaria.

Un análisis de este fenómeno permitiría la visibilización del impacto que esto tiene en la economía de las mujeres, y cómo este entramado se transforma en una manifestación de la violencia patrimonial hacia ellas, una negación de los derechos de la niñez y la adolescencia, y la falta de profundas transformaciones culturales, las mismas que dificultan la comprensión de que la vulneración de este derecho afecta el acceso a la educación de los hijos e hijas y repercute en el ámbito cultural, perpetuando patrones socioculturales que justifican este comportamiento, lo cual significa un reto en términos del avance de una educación no sexista e inclusiva que pueda plantear nuevos paradigmas que reafirmen la paternidad responsable en la región.

\section{Referencias}

Amorós, C. (1990). Violencia contra las mujeres y pactos patriarcales. En V. Maquieira y C. Sánchez (Eds.), Violencia y sociedad patriarcal (pp. 1-15). Pablo Iglesias. 
Amorós, C. (1987). Espacio de los iguales espacio de las idénticas. Notas sobre el poder y principio de individualización. Revista Arbor: Ciencias, Pensamiento y Cultura, 504, 113-128.

Arroyo, R. (2007). Derechos humanos de las mujeres en América Latina: Un camino para la transformación cultural. Revista Pensamiento Jurídico Feminista. Deconstruir, 3, 89-98.

Arroyo, R. (2002). Las normas sobre violencia contra la mujer y su aplicación, un análisis comparado para América Central. CEM-MUJE-IEM.

Bonino, L. (2004). Los micromachismos. Revista la Cibeles Ayuntamiento de Madrid, 2, 1-6.

Bourdieu, P. (1999). La dominación masculina. Anagrama.

Cillero, M. (2007). El interés superior del niño en el marco de la Convención Internacional sobre los Derechos del Niño. En UNICEF (Ed.), Justicia y Derecho del Niño (pp.125-142.). UNICEF.

Cobo, R. (1995). Género. En C. Amorós (Ed.), 10 palabras clave sobre la mujer (pp. 55-84) Editorial Verbo Divino.

Cobo, R. (1996). Discriminación positiva: El largo camino hacia la igualdad. Revista Crítica, 831, 2628.

CEDAW. (1992). Recomendación General número 19. Violencia contra la mujer. CEDAW.

CEDAW. (2004). Recomendación General Número 25 sobre el párrafo 1 del artículo 4 de la Convención sobre la eliminación de todas las formas de discriminación contra la mujer, referente a medidas especiales de carácter temporal. CEDAW.

CEDAW. (2010). Recomendación general número 28 relativa a las obligaciones básicas de los Estados partes de conformidad con el artículo 2 de la Convención sobre la eliminación de todas las formas de discriminación contra la mujer. CEDAW.

CEDAW. (2015). Recomendación General Número 33 sobre acceso a la justicia de las mujeres. CEDAW.

Eicheler, M. (1999). Cambios familiares: familias, políticas e igualdad de género. En A, Facio y L, Fries (Eds.), Género y derecho (pp. 443-485). La Morada.

Facio, A. (1992) Cuando el género suena cambios trae. Una metodología para el análisis de género del fenómeno jurídico legal. ILANUD.

Flores, S. y Lindo, P. (2005). Pautas conceptuales y metodológicas. UNIFEM/PNUD.

García, R. (2012). La educación desde la perspectiva de género. Revista ENSAYOS, 27, 1-18.

Huaita, M. (1999). Desigualdades de género en las consecuencias económicas financieras del divorcio. En A. Facio y L. Fries (Eds.), Género y derecho (pp. 487-539). La Morada.

Kelly, L. (1987). Surviving sexual violence. Polity Press.

Lagarde, M. (1997). La construcción de los sujetos. En V. Castillo y P. Orosco (Eds.), Claves feministas para el poderío y la autonomía de las mujeres (pp. 59-136). Managua.

Lagarde, M. (2005). Para mis socias de la vida. horas y HORAS la editorial.

Lagarde, M. (2014). El feminismo en mi vida. Hitos, claves y topias. horas y HORAS la editorial.

Ordeñana, T. y Barahona, A. (2016). El derecho de familia en el nuevo paradigma constitucional. Cevallos Editorial.

Pazmiño, E. (2018). Introducción. En M. Romo, Código de la niñez y la adolescencia (pp. 7-13). DPE. 
Pedone, C. (2010). Cadenas y redes migratorias: Propuesta metodológica para el análisis diacrónico-temporal de los procesos migratorios. EMPIRIA Revista de Metodología de las Ciencias Sociales, 19, 110-132. https://doi.org/10.5944/empiria.19.2010.2016

Romo, M. P. (2018). Código de la niñez y la adolescencia. DPE

Rubin, G. (1989). Thinking sex: Notes for a radical theory of the politics of sexuality. En C. S. Vance (Ed.), Pleasure and danger: Exploring female sexuality (pp. 57-89). Pandora.

Saltman, J. (1988). Equidad y género. Una teoría integrada de estabilidad y cambio. Cátedra.

Sánchez O. (1988). Anotaciones acerca del modelo de socialización patriarcal. En M. Laverde y H. Sánchez (Eds.), Voces insurgentes (pp. 208-224) Editorial Guadalupe.

Tortonda, A., Barriga, E. y Gonzáles, R. (2019). Hacia una escuela inclusiva: perspectiva de género y literatura. Revista de Educación Inclusiva, 3(1), 66-76.

UNESCO. (2005). Orientaciones para la inclusión. UNESCO.

\section{Breve CV de la autora}

\section{Roxana Arroyo}

Doctorado en Derechos Humanos por la Universidad Carlos III de Madrid "Instituto Bartolomé de las Casas”. Profesora titular del Instituto de Altos Estudios Nacionales, La Universidad de Posgrado de Ecuador, IAEN. ORCID ID: https://orcid.org/oOOO-OOO2o215-6709. Email: roxana.arroyo@iaen.edu.ec 